INVESTIGACIÓN

Recibido: 1/10/2013

Revisado: 1/11/2013

Aprobado: 1/12/2013

\title{
EL LENGUAJE COMO INSTRUMENTO TÉCNICO EN JOSÉ GAOS
}

\author{
PEDRO PABLO RIVAS OSORIO \\ Centro de Estudios e Investigaciones Latinoamericanas CEILAT \\ Universidad de Nariño
}

\section{RESUMEN}

Lo que el texto pretende, es dejar en evidencia el acto en el que el lenguaje funge como articulador entre el sujeto, el acto o actividad de proferir el concepto y las relaciones que de este acto se derivan; en la lógica de José Gaos, la percepción y la comprensión.

La ruta señalada para encontrar la percepción y la comprensión, se garantiza por el momento del "anticipo" en lo que el texto expone como una explicación o idea de sí mismo para que los participantes tengan una idea acerca de su asistencia o encuentro con el lenguaje que enuncia al sujeto, al concepto y las relaciones que de esto se derivan (Filosofía de la filosofía). El texto en definitiva, nos hace recordar una de las propiedades fundamentales del filosofar; todas las proposiciones que esta acción enuncia pueden y deben aplicarse al sujeto que la anuncia. El lenguaje en su versión "técnica" como "fenomenología de la expresión verbal" vuelve inteligible y coherente el devenir de la filosofía por la filosofía.

Palabras clave: Filosofía, Intelegibilidad, Discurso, Fenomenología.

\section{ABSTRACT}

What the text intends is to make evident the act in which language functions as an articulator between the subject, the act or activity of uttering the concept and the relationships that derive from this act; In the logic of José Gaos, perception and understanding.

The route indicated to find the perception and the understanding, is guaranteed by the moment of the "anticipation" in what the text exposes like an explanation or idea of itself so that the participants have an idea about its attendance or encounter with the language Which enunciates the subject, the concept and the relationships that derive from this (Philosophy of philosophy). The text, in short, reminds us of one of the fundamental properties of philosophizing; All the propositions that this action states can and should be applied to the subject who announces it. The language in its "technical" version as "phenomenology of verbal expression" renders intelligible and coherent the becoming of philosophy by philosophy.

Key words: Philosophy, Intelegibility, Speech, Phenomenology. 
"Este curso va a ser un curso de Filosofía de la Filosofía (El subrayado indica que en la obra escrita"1 de Gaos se resalta el texto).

Así introduce el curso De la Filosofía. En ella parece se señala inmediatamente el contenido del curso: Se trata de una filosofía que va dar razón de la filosofía. Es un discurso cuyas características son el rigor y la intelelegibilidad; "es una teoría o un dar razón teórica de la filosofía" con una característica determinante: Esta teoría "Es filosofía".

Gaos indica que hay "Idea de la Filosofía de la Filosofía del curso". Es decir que el curso que se va a desarrollar tiene una filosofía y no es más que una anticipación del discurso sin el "rigor" y la "intelegibilidad" del mismo, con lo cual nos esta dando a entender que la Filosofía de la Filosofía debe desarrollarse a partir de si misma, pues cualquier forma de hablar de la misma diferente a ella seria de alguna manera una forma de desfigurar su discurso, alejarse del rigor y de la intelegibilidad del mismo. Aquí nos muestra Gaos que la Filosofía de la Filosofía es un discurso riguroso e inteligible. Caracterizamos a la filosofía de la Filosofía como un discurso que tiene rigor y que es inteligible. La Filosofía de la Filosofía es Filosofía. Es "un dar razón teórica de la Filosofía y ella misma es filosofía".

La idea que tiene Gaos de lo que es la Filosofía de la Filosofía la podemos entresacar del curso que desarrolla con el titulo De la Filosofía y que no es más

1. GAOS, José. Filosofía de la Filosofía e Historia de la Filosofía. 2 Ed. UNAM. Mexico, 1987. que la Filosofía de la Filosofía; a pesar de su afirmación de que no es sino un "curso de la Filosofía de la Filosofía". Encontramos en Gaos algo que le ha caracterizado: su actitud pedagógica. Señala que "curso no se trata de Filosofía de la Filosofía, simplemente; sino de un curso de Filosofía de la Filosofía" Gaos se ajusta a la "tradición" en el hecho de que a todo curso debe exigírsele por parte de los asistentes un anticipo, una explicación, una "idea de sí mismo" para que los participantes "posibles asistentes a ellos" tengan un conocimiento "de causa acerca de su asistencia efectiva a ellos". De nuevo afirmamos lo que ya dijimos anteriormente: en este caso el "rigor y la inteligibilidad" frenan el discurso de la filosofía, son remplazados "por la tradicional y razonada exigencia de los cursos".

Nos detenemos aquí para considerar lo necesario. Gaos anota que la explicación de lo que va a ser el curso - requiere "un resumen anticipado, que como tal requerirá explicaciones en las que vendrá a consistir la Filosofía de la Filosofía del curso mismo. Es interesante no solamente desde el punto de vista pedagógico, propedéutico, la indicación que hace Gaos de que éste resumen por anticipado se convierte en $\mathrm{La}$ Filosofía de la Filosofía del curso mismo. Parecería que está afirmando que esta anticipación deje en el ponente y en el participante del curso una idea acerca de lo que se va a desarrollar en dicho curso, pero diferente a lo que el mismo curso será. Quisiera anotar que ya Hegel a desarrollado en el prologo a La Fenomenología del Espíritu (las teorías científicas del presente) el tema en relación a todo anticipo que se hace de 
un texto escrito; o en el caso que nos ocupa lo asimilamos a la tradicional y razonada exigencia de la presentación inicial de un curso en el que se combina el ejercicio de la escritura y la oralidad:

"Parece que, en una obra filosófica, no solo resulta superfluo, sino que es incluso, en relación a la naturaleza misma de la cosa, inadecuado y contraproducente el anteponer, a manera de prólogo y siguiendo la costumbre establecida, una explicación acerca de la finalidad que el autor se propone en ella acerca de sus motivos y de las relaciones que entiende que su estudio guarda con otros anteriores o coetáneos en cuanto al mismo tema. En efecto lo que sería oportuno decir en un prólogo acerca de la filosofía - algo así como una indicación histórica con respecto a la tendencia y el punto de vista, al contenido general y a los resultados, un conjunto de afirmaciones y aseveraciones sueltas y dispersas acerca de la verdad - no puede ser verdadero en cuanto al modo y la manera en que la verdad filosófica debe exponerse"2

En esta extensa nota de Hegel que he traído podríamos de pronto descubrir que Gaos de alguna manera extrapola a la explicación oral del curso, el cual ha de aparecer como obra escrita, el sentido de las afirmaciones hegelianas; así como en el prólogo a la obra escrita aparecerían una serie de elementos sueltos y dispersos la explicación oral del curso, al iniciar el mismo, quitaría el rigor e inteligibilidad de lo que se va a desarrollar; es decir, aceptamos de pronto afirmar que reconoce la afirmación de Hegel de que la filosofía o el saber filosófico se expone, se desarrolla.

2. HEGEL, G. F. La Fenomenología del Espíritu. F. C. E. 5a Reimpresión. México, 1982. Prólogo, p. 8.
Podríamos también indicar que entendemos "la Filosofía de la Filosofía del curso" en sentido estricto como aquello que se va a desarrollar en el transcurso de la totalidad del curso lo cual significa que la Filosofía de la Filosofía como teoría de la filosofía abarca mucho más, el concepto abarca mucho más de lo que podría desarrollarse durante el tiempo de duración del curso; es decir, de pronto Gaos nos está indicando que el mismo concepto de filosofía, según su manera de entenderlo, se desarrolla en la producción filosófica que se da. Esto lo podríamos analizar articulando con planteamientos que hace Gaos sobre la relación entre historia de la filosofía y la filosofía.

Volvamos a la obra escrita de Gaos: "La Filosofía de la Filosofía debe empezar ex-abrupto". La exposición de Gaos empezará por una fenomenología de la expresión verbal.

La Filosofía de la Filosofía. Ya hemos indicado como para Gaos es un dar razón de la filosofía, es una teoría de la filosofía, y es filosofía, de lo anterior se desprende entonces que la Filosofía de la Filosofía es un filosofar acerca, sobre la Filosofía. Este filosofar se vuelve filosofía y tiene como objeto al hombre mismo. Esto hace aparecer una relación recíproca en el sentido de razón de la filosofía por el hombre y dar razón del hombre por la filosofía. En palabras de Gaos: se trata de dar razón de la filosofía por el hombre y del hombre por la filosofía; no es una antropología filosófica o filosofía del hombre, sino filosofía: "un dar razón de la filosofía por el hombre y de éste por la filosofía, de las categorías por la historia y de ésta por las categorías -en una circularidad de la que habrá a su vez que dar razón- 
que debería representar la resolución de los problemas planteados" (Leción I). Aquí detengamos. Surge la pregunta "qué es la filosofía" en el pensamiento de Gaos. Al finalizar el curso en "la autobiografía y autocrítica del curso" nos da indicaciones que nos permitirán una aproximación:

"Esta autocrítica no debe concluir más que de la manera siguiente:

La Filosofía de la Filosofía del curso ha concluido que toda filosofía es en conjunto subjetivo - o válida únicamente para su sujeto, o su autor. Consecuente conmigo mismo, como válido para ustedes ni para nadie; no puedo más que considerar como una exposición de mi "perspectiva" que no puede ser compartida por nadie más que en la proporción que sea idéntico conmigo mismo. Por lo tanto, no espero el asentimiento de ustedes, sino justamente el disentimiento - que es lo único que puede confirmase en mi perspectiva, por la paradójica dialéctica de la unidad y pluralidad de los sujetos que yo reduciría una vez más, a la antinomia: La Filosofía de la Filosofía del curso es una teoría del tropo de agripa, de la discrepancia de los filósofos: quien discrepe de ella, la confirma: quien asintiere a ella, la invalidaría - espero, con arreglo a ella misma, asentir a ella no puede nadie más que yo.

Así que no se empeñen ustedes en asentir a ella - por llevarme la contraria de la contraria: ni queriendo asentir a ella, pueden hacer, ni harán de hecho, salvo, si les divierte, verbalmente, más que disentir de ella.

En la medida en que yo mismo no soy idéntico conmigo mismo, sino que yo voy viviendo y pensando diferente - o soy infinito, yo mismo he pensado y pensaré $-\mathrm{y}$, naturalmente, he vivido $\mathrm{y}$ viviré deficientemente, y al percatarme, por obra del mismo vivir y pensar diferente, he disentido y disentiré de mi. Tal es el fondo último de la subjetividad y momentaneidad. Con que, que otra cosa van a ser ustedes, mis pobres amigos y enemigos por eso no hay diálogo filosófico - fondo: filosóficamente, a fondo no puede haber más que el monólogo en soledad. No es mi culpa, ni por ende mío el remedio - imposible, de que esto se reduzca al absurdo los congresos de filosofía - de la misma enseñanza de la filosofía".

Rápidamente podríamos afirmar que la perspectiva de Gaos frente a la filosofía es que ésta es subjetiva, válida únicamente para su sujeto o su autor, y no hay diálogo filosófico profundo, a fondo, que la filosofía no es más que un monólogo, etc., etc. En términos generales podríamos acaso pensar que la pregunta "qué es la Filosofía" ya señala en su respuesta un sentido, ya indica un significado, ya nos está hablando o testimoniando algún indicio sobre el sujeto que interroga, sobre su personalidad. A la ligera podríamos decir que lo anteriormente traído invalida no solamente el curso que se ha desarrollado sino la filosofía, la teoría de la filosofía que se ha desarrollado; en general es la invalidación de toda relación comunicativa. Nos parece que hay que hacer diferentes tipos de lecturas sobre los textos del autor que hemos venido tratando; señalaría dos en este momento: uno aquel en el que el filósofo habla de sí mismo, de lo que es, de su personalidad, de sus preocupaciones, en una palabra de sus avatares, de su autobiografía. El otro nivel es el de lenguaje filosófico, el del categorial, aquel que es en sentido estric- 
to filosofía como producción del sujeto que piensa, que produce saberes, de ahí que, el mismo Gaos a continuación de lo anteriormente dicho continua afirmando: "A pesar de su subjetividad en conjunto, también en conjunto aunque bajo otro punto de vista, este curso ha sido un resultado y una síntesis biográficos de la historia contemporánea de la filosofía: "esto se ha extendido de Husserl a Heidegger... y los afines de sus afines han sido sino temas para los técnicos de las escolásticas, de las que la última ha sido la fenomenológica".

Lo que venimos afirmando nos induce a descentralizar el análisis en lo autobiográfico y centralizarlo en otro tipo de espacio, en el análisis de los conceptos, de las categorías, de la teoría: en lo que Gaos denomina en sentido estricto "Filosofía".

La pregunta por la filosofía implica la pregunta por el sujeto que filosofa y por su situación vivencial; también incluye la pregunta, o la historia misma de la pregunta, en esa pregunta confluyen entonces la vida de quien interroga, el por qué de su interrogación y sus esfuerzos por dar la respuesta; pero esas respuestas tienen como materia primaria además de la situación vital del filosofo, la historia misma de la filosofía, de la pregunta, de las respuestas. Aquí pensamos nosotros se rasga, se hace ruptura con el punto de vista autobiográfico sobre la filosofía; de aquí que ella comience exabrupto por una fenomenología de la expresión y lo normal es que las expresiones son expresiones producidas por los sujetos y en este caso por el sujeto filosofo; en esas expresiones se conjugan todos los elementos que componen las situaciones del sujeto.

Esta situación ¿no es acaso la vida cotidiana en sus diversas formas de manifestarse tanto socialmente como en el sujeto que piensa? Nos preguntamos.

La Filosofía de la Filosofía entonces implica una concepción histórica de la misma y una perspectiva del sujeto sobre la misma. De esta manera desde el punto de vista histórico, de la historia, no hay filosofías verdaderas o falsas, sino que son momentos del desarrollo de la filosofía; así la realidad, su estructura se da de diferente manera según el momento histórico y el momento por el cual el sujeto que piensa está pensando. No tenemos ninguna duda en afirmar que esta forma de ver la filosofía en sentido estricto y las filosofías nos llevan entonces a pensar en la relación y diferencia que hay entre la filosofía y las filosofías. Gaos afirma "la realidad se da así, la unidad de una pluralidad de realidades para cada uno de nosotros - en el que para cada uno expresa la pluralidad de realidades y el de nosotros la unidad de ésta pluralidad". Aquí aparece el sujeto como individualidad y se remplaza por el nosotros; continúa Gaos afirmando:

"conclusión: en la medida en que la Filosofía sea cuerpo de proposiciones acerca de la realidad en general, esto es, en su unidad, que significa aquí su integridad, justamente ha de fracturarse, pues en tantos cuerpos de proposiciones, en tantas filosofías como sujetos que piensen tal cuerpo de proposiciones como filósofos- [más adelante añade:] "por esta vía se arriba a la idea de que 
en la medida en que la filosofía sea un cuerpo de proposiciones sobre la realidad en su integridad, u expresión de la realidad para un sujeto se acercará a ser pensamiento personal, que variará según otra dimensión todavía: la de la vida del sujeto mismo"3.

Aquí encontramos que el objeto de la filosofía es la que define, éste objeto no es más que la realidad como totalidad y las filosofías al referirse a éste objeto, a la totalidad de la realidad, tiene una unidad precisamente en ese objeto; el hecho de que el objeto se presente bajo la perspectiva del sujeto filósofo permite la diversidad de filosofías. Parecería que hay una contradicción en Gaos entre la multiplicidad de filosofías y la historia de la filosofía. La historia de la filosofía nos diría una serie de elementos sacados de estas filosofías los cuales, dichos elementos, serían la estructura fundamental, los conceptos básicos, las categorías que constituirían la filosofía; no otra cosa hace Gaos cuando en el texto que estamos analizando de la filosofía identifica conceptos y categorías básicas como estructuras del hombre que el denomina en la lección primaria del curso: "conceptos principales" o "categorías". De ahí que Gaos empieza o la filosofía según Gaos empieza ex-abrupto con la fenomenología de la expresión verbal. La fenomenología de la expresión verbal se justifica, lo podemos afirmar, a partir de la cita anteriormente traída en el hecho de que la filosofía nos aparece contem-

3. GAOS. Sobre La Filosofía de la Filosofía, recogido en el libro Filosofía e Historia de la Filosofía, 2 ed. UNAM. México 1987, pp: 60 - 67 (primera ed. Editorial Stylo. 1947.) poráneamente e históricamente como un conjunto de expresiones verbales, de proposiciones. Gaos, partiendo de la fenomenología de la expresión verbal, penetra en los conceptos principales de la filosofía, desarrollados en la historia de la filosofía; estos los caracterizan a la filosofía. Esta manera de tratar Gaos la filosofía nos remite a Kant.

Según Gaos "el método fenomenológico prescribe partir de expresiones verbales designantes del objeto de que se quiere hacer la fenomenología y de un ejemplo o ejemplar de tal objeto (Lección II - "Una expresión verbal"). Parte de la expresión verbal "pero no de esta forma general del lenguaje "sino de una singularidad: "esta sala" -. Esta expresión no es filosófica, pero es una tipificación de lo que es "una expresión verbal" y permite describir un "fenómeno total de expresión verbal en el que hay que distinguir todo lo siguiente poniéndole por nombres técnicos, según prescribe también el método" (Lección II).- Esta terminología técnica se debe caracterizar por tener "coherencia" y se precisa; lo que hace que la descripción sea rigurosa e inteligible.

La relación entre el pensar y la expresión verbal es manifiesta del pensamiento; se impone la necesidad de analizar el lenguaje en cuanto expresión verbal y el acto total o fenómeno total en que ella está suscrita. En este fenómeno hay una situación en la cual hay expresividad: en ella interviene el sujeto, el acto o actividad de proferir, el concepto, relaciones entre momentos, la percepción y la comprensión (Gaos señala catorce "ingredientes" en el "fe- 
nómeno total"). En la expresión verbal como tal se puede identificar elementos constitutivos de la misma: la expresión en el sentido estricto estructurada por sonidos, grafos o elementos físicos, el concepto o lo expresado, los actos subjetivos de los sujetos que intervienen como mociones y emociones; del objeto designado.

Trataremos de enunciar muy someramente los múltiples elementos que intervienen en el "fenómeno total" de la expresión verbal:

1. "Lo expresante".- Expresa el objeto.- 1.1. -La expresión verbal. -1.2 - El sujeto. 1.2.1. El Sujeto Concreto. 1.2.2. El Sujeto Destinatario.-

1. Lo "expresante". Expresan el objeto.-

1.1 La expresión verbal: sonidos articulados; tono enunciativo.

1.2 Expresión escrita: para mí como sujeto que escribe; signos gráficos; como posibilidad para otros.

\section{ELEMENTOS:}

a.- Sonidos articulados y sus signos; "lo notificante" y "lo designante".

b.- Relaciones; o sonidos o signos - con el objeto: "lo designante" tono enunciativo y sus signos: "lo significante".

1.2. El Concepto (esta sala): "lo notificado", "lo objetivante". Hecho psíquico que media entre el objeto (esta sala) y los "sonidos articulados".

De esta manera los sonidos o los grafos "expresan" el concepto 'esta sala' y este expresa el objeto percibido esta sala.

Concepto: 'esta sala'. Objeto: esta sala. Expresión: "esta sala".

2. El objeto

2.1. El objeto: 'esta sala'; lo objetivado: esta sala Relaciones: el objeto en relación a los sonidos o signos: "므 designado".

3. El sujeto.

El sujeto psíquico aparece necesariamente por la "actitud o estado afectivo", por su naturaleza psíquica, la que necesariamente o por naturaleza, es de un objeto psíquico; por otra parte el sujeto aparece porque es un sujeto el registrador de los sonidos, el tono y los signos; además de ser él el que expresa el concepto: "efectivamente en el fenómeno total de expresión verbal de que es centro la expresión "esta sala", hay un sujeto psíquico que es a una el que viene profiriendo la expresión oral tantas veces como había escrito la escrita, el del concepto 'esta sala' y el de la actitud o estado de ecuánime contemplación de esta sala misma; este sujeto "-soy yo; quien lee ahora aquí, en esta sala" (Lección II).

3.1. El sujeto "concreto" - (soy yo afirma Gaos) - es el sujeto; en el se da la unidad de todos los elementos que componen la expresión verbal y que son relativos a este sujeto "concreto". Se la denomina simplemente sujeto; es:

a. "El expresante" de la expresión verbal.

b. "El profiriente" o "el emitente" de los sonidos y su tono. 
c. "El escribiente" de los signos gráficos.

d. "El notificante" del concepto.

e. "El objetivante" por mediación del concepto del objeto.

f. "El designante" del objeto (emite sonidos), tonos signos)

g. "El significante" de su propio estado o afección.

3.2. El destinatario.

El destinatario sujeto a quien va dirigida la expresión oral es sujeto psíquico. Es sujeto de la cual se presupone ser capaz de percibir y comprender la expresión oral con la configuración que le ha dado el sujeto concreto o sujeto no destinatario. Los destinatarios son "sujetos percipientes y que comprenden".

El destinatario en su actitud de destinatario esta:

a. "Percibiendo": (percibe) auditivamente, acústicamente sonidos, tono; visualmente, ópticamente signos, grafos...

b. "Comprendiendo": (comprende) el concepto notificado, en el objeto, la ecuanimidad...

\section{BIBLIOGRAFÍA}

GAOS, José. Filosofía de la Filosofía e Historia de la Filosofía. 2 Ed. UNAM. México, 1987.

GAOS. Sobre La Filosofía de la Filosofía, recogido en el libro Filosofía e Historia de la Filosofía, 2 Ed. UNAM. México 1987 (Primera Ed. Editorial Stylo. 1947).

HEGEL, G. F. La Fenomenología del Espíritu. F. C. E. 5a Reimpresión. México, 1982. Prólogo. 